The Astrophysical Journal, 238:829-841, 1980 June 15

() 1980. The American Astronomical Society. All rights reserved. Printed in U.S.A.

\title{
THE H II REGION-MOLECULAR CLOUD COMPLEX W 3: A MODEL DERIVED FROM MOLECULAR OBSERVATIONS
}

\author{
HÉLÈnE R. DICKEL \\ Astronomy Department, University of Illinois; and Sterrewacht, Leiden \\ Received 1979 August 8; accepted 1979 December 11
}

\begin{abstract}
A model of the W 3 molecular cloud is constructed from the analysis of ${ }^{12} \mathrm{CO},{ }^{13} \mathrm{CO}, \mathrm{CS}, \mathrm{HCN}$, and $\mathrm{H}_{2} \mathrm{CO}$ molecular data. Originally an initial cloud of mass $\sim 5 \times 10^{4} M_{\odot}$ started to fragment and collapse after passage through the spiral density-wave shock of the Perseus arm. The open cluster $(\mathrm{Ocl} 352)$ which excites the present W4 H II region formed at the head of the cloud a few million years ago. The ionization front with its associated shock is expanding into the remaining W3 molecular cloud where its effects are seen in the velocities of W3(OH) and the infrared source AFGL 333. The bright dense "core" of the W3 molecular cloud which contains six embedded compact $\mathrm{H}$ II regions, eight near-infrared sources, and three $\mathrm{H}_{2} \mathrm{O}$ masers is collapsing and possibly rotating.

The shock front of the expanding optical H II region, IC 1795, is moving into the edge of the W3 molecular core at $2-3 \mathrm{~km} \mathrm{~s}^{-1}$, as evidenced by the motion of the $\mathrm{CO}$ gas behind the shock front. However, this shocked gas represents only a small fraction of the total material present, so the shock cannot be responsible for triggering the collapse of the whole W3 core.

The abundance of CS is probably a factor of at least 10 lower in the direction of the embedded complex of near-infrared sources designated 5,6 , and 7 than at positions only $0.6 \mathrm{pc}$ away. Several possible explanations are explored: the most likely involves ion-molecule chemistry.
\end{abstract}

Subject headings: interstellar: molecules — nebulae: abundances — nebulae: general nebulae: individual

\section{INTRODUCTION}

In the previous paper (Dickel et al. 1980, hereafter $\mathrm{D}^{2} \mathrm{WW}$, the distributions of $\mathrm{CO}, \mathrm{CS}$, and $\mathrm{HCN}$ emission from the W3 cloud were presented. In this paper the data are further analyzed and a model of the region is developed. The model must account for a large variety of interrelated features which are identified in Table 4 of the earlier paper $\left(\mathrm{D}^{2} \mathrm{WW}\right)$. Throughout this paper, I shall refer to the designations and properties of the objects as given in that table. The most important observational features can be summarized as follows:

1. The intensities and widths of the molecular lines are larger in the direction of embedded excitation sources such as masers, IR sources, and compact H II regions (e.g., the W3 core shown in Fig. 1a).

2. There are three separate regions with distinct velocities. The brightest part of the W3 molecular cloud, the W3 core, has a $V_{\mathrm{lsr}} \sim-40.5 \pm 2.5 \mathrm{~km} \mathrm{~s}^{-1}$; the region along the $\mathrm{W} 4$ ionization front has a $V_{\mathrm{lsr}} \sim$ $-47 \pm 2 \mathrm{~km} \mathrm{~s}^{-1}$; and the remaining, less-intense part of the W3 cloud has a $V_{\mathrm{lsr}} \sim-43 \pm 1 \mathrm{~km} \mathrm{~s}^{-1}$. The separation of the first two regions is delineated by the prominent north-south dust lane which runs between W4 (IC 1805) and W3 (IC 1795). ${ }^{12} \mathrm{CO}$ emission is detected at both $-46 \mathrm{~km} \mathrm{~s}^{-1}$ and $-40 \mathrm{~km} \mathrm{~s}^{-1}$ along this boundary (as shown in Fig. 2).
3. Emission from dust in the far-IR and at $1 \mathrm{~mm}$ plus emission from $\mathrm{CO}, \mathrm{CS}$, and $\mathrm{HCN}$ are found in the W3 core.

4. The ${ }^{12} \mathrm{CO}$ and $\mathrm{CS}$ profiles are asymmetric throughout the bright, dense core.

5. In the direction of IRS 5-6-7 where the $1 \mathrm{~mm}$ dust emission has its maximum, there is clear self-reversal in the center of the ${ }^{12} \mathrm{CO}$ profile. The position of the selfabsorption dip relative to the ${ }^{13} \mathrm{CO}$ peak indicates that the foreground material which is just becoming optically thick is moving away from the observer by a few $\mathrm{km} \mathrm{s}^{-1}$ relative to the bulk of the material which forms the line core.

6. The $\mathrm{HCN}(J=1-0, F=2-1)$ line is wide and possibly self-absorbed toward IRS 5-6-7, whereas the CS $(J=2-1)$ line is weak there; elsewhere the $\mathrm{HCN}$ and CS lines have similar intensities.

7. There is a velocity gradient across the $\mathrm{W} 3$ core from $\sim-38 \mathrm{~km} \mathrm{~s}^{-1}$ in the SE near the border with the optical $\mathrm{H}$ II region IC 1795 to $-43 \mathrm{~km} \mathrm{~s}^{-1}$ in the NW. (The mean effect is along a line which passes close to the three $\mathrm{H}_{2} \mathrm{O}$ masers in the list of Genzel and Downes 1977).

8. Lines of constant velocity run parallel to the edge of the optically visible diffuse $\mathrm{H}$ il region (the main part of IC 1795) with the more positive velocities near this edge and less on both sides. 
9. There is a sequence of ages from a few $\times 10^{6}$ years for the OB cluster exciting the W4 $\mathrm{H}$ II region (e.g., Ishida 1969), through to a few $\times 10^{5}$ years for the formation of the protostar(s) IRS 5 (Genzel and Downes 1977), down to $\sim 10^{4}$ years for such events as the emergence of the compact $\mathrm{H}$ II region $\mathrm{W} 3 \mathrm{~A}$ out of the dense molecular cloud (Dickel and Harten 1980).

In $\S$ II a model is developed for the heirarchy of components, starting with the overall cloud and working inward to the smaller components, incorporating additional effects, as needed, to explain them. A final summary and conclusions appear in § III.

\section{ANALYSIS OF THE MOLECULAR DATA TO DEVELOP A MODEL OF W3}

\section{a) Overall Cloud}

\section{i) ${ }^{13} \mathrm{CO}$ Column Density and the Total Mass}

Under the assumption of local thermodynamic equilibrium, the ${ }^{13} \mathrm{CO}$ column density determined from the observed ${ }^{12} \mathrm{CO}$ and ${ }^{13} \mathrm{CO}$ brightness temperatures is, in general, a good approximation to the true column density in dark clouds except in the outer, low-density areas where the true column density may be a factor of 4 or more higher (Dickman 1976, 1978). These conclusions were confirmed in this research for a giant molecular cloud which contains excitation sources, using radiative transfer calculations similar to Dickman's. It was found that the LTE column densities should be correct for regions with molecular hydrogen densities $\gtrsim 10^{3} \mathrm{~cm}^{-3}$ and kinetic temperatures greater than about $20 \mathrm{~K}$, which corresponds to an observed ${ }^{12} \mathrm{CO}$ temperature near $15 \mathrm{~K}$. Therefore, in the $\mathrm{W} 3$ core and $\mathrm{W} 3(\mathrm{OH})$ where the ${ }^{13} \mathrm{CO}$ line is reasonably strong, accurate column densities are obtainable. However, for observed ${ }^{12} \mathrm{CO}$ temperatures in $\mathrm{W} 3$ between 5 and $15 \mathrm{~K}$, the ${ }^{13} \mathrm{CO}$ column densities (derived assuming LTE) could be a factor 1.1-2 too low. The results for the W3 core are plotted in Figure $1 a$. The ${ }^{13} \mathrm{CO}$ column density has a maximum value of $16 \times 10^{16} \mathrm{~cm}^{-2}$ in the direction of the $1720 \mathrm{MHz}$ OH maser, falls to $1 \times 10^{16} \mathrm{~cm}^{-2}$ just outside the $10 \%$ contour of the $1 \mathrm{~mm}$ dust emission (see Fig. 3 of $\mathrm{D}^{2} \mathrm{WW}$, with its reference to Westerbrook et al. 1976), and drops to less than 0.5 $\times 10^{16} \mathrm{~cm}^{-2}$ over most of the southern group of compact $\mathrm{H}$ II regions and the diffuse $\mathrm{H}$ II region IC 1795 to the east. In fact, the contours of $N\left({ }^{13} \mathrm{CO}\right)$ resemble those of the $1 \mathrm{~mm}$ emission except that the latter peaks on IRS 5-6-7 rather than at the position of the $1720 \mathrm{OH}$ maser.

The mass of the molecular cloud can be calculated from the ${ }^{13} \mathrm{CO}$ column densities once the distance and the abundance ratio of $\mathrm{H}_{2}$ to ${ }^{13} \mathrm{CO}$ are estimated. For a distance of $2 \mathrm{kpc}$ (Ogura and Ishida 1976) and a relatively low $\left[\mathrm{H}_{2}\right] /\left[{ }^{13} \mathrm{CO}\right]$ ratio of $4 \times 10^{5}(\mathrm{e} . \mathrm{g}$., Dickman 1978) a minimum mass of $5 \times 10^{3} M_{\odot}$ is found for the region of the W3 core enclosed by the
$N\left({ }^{13} \mathrm{CO}\right) \sim 10^{16} \mathrm{~cm}^{-2}$ contour in Figure $1 a$ (which corresponds to $T_{A}{ }^{*} \geq 15 \mathrm{~K}$ on Fig. $2 a$ in $\mathrm{D}^{2} \mathrm{WW}$ ). The mass estimates become less certain outside $T_{A}{ }^{*}=15 \mathrm{~K}$ because of the poor signal-to-noise ratio of the ${ }^{13} \mathrm{CO}$ measurements and the breakdown of the LTE assumption, but estimates for the minimum mass for the three regions with $T_{A}{ }^{*} \geq 10 \mathrm{~K}$ are $\sim 8 \times 10^{3} M_{\odot}$ for the W3 core, $\sim 4 \times 10^{3} M_{\odot}$ for $\mathrm{W} 3(\mathrm{OH})$, and $\sim 8$ $\times 10^{3} M_{\odot}$ for AFGL 333. For comparison, the total mass of just the known $\mathrm{O}$ and $\mathrm{B}$ stars exciting W4 is estimated from the spectral types (Ishida 1969) with their corresponding masses (Allen 1973) to be $10^{3} M_{\odot}$. This is a minimum value since these 79 stars were assumed to be main-sequence stars unless specified otherwise by Ishida. The rest of the low-density gas mapped by Lada et al. (1978) contributes $\sim 3$ $\times 10^{4} M_{\odot}$ or up to $60 \%$ of the total mass of the W3 molecular cloud. If the $\left[\mathrm{H}_{2}\right] /\left[{ }^{13} \mathrm{CO}\right]$ ratio is closer to $10^{6}$, which may be the case (see Table 2), and if the $N\left({ }^{13} \mathrm{CO}\right)$ in the outer parts was underestimated by a factor of 4 , then the total mass of the W3 cloud would increase from $\sim 5 \times 10^{4} M_{\odot}$ to $\sim 4 \times 10^{5} M_{\odot}$. A mass of $\sim 4 \times 10^{4} M_{\odot}$ is needed to bind the outer parts of the cloud with an extent of $100 \mathrm{pc}$ against disruption by the tidal force of the galaxy at a galacto-centric distance of $11.5 \mathrm{kpc}$ (see Figs. 2 and 3 in the paper by Stark and Blitz 1978). Thus the original W3 molecular cloud was able to hold together until it entered the Perseus spiral shock (see $\S$ II $a[$ iv $]$ ).

\section{ii) The Location of Major Components}

In the preceding paper $\left(\mathrm{D}^{2} \mathrm{WW}\right)$ we concluded that the W3 molecular cloud and the W4 $\mathrm{H}$ II region are in physical contact based on the common distance of $\sim 2 \mathrm{kpc}$ for the exciting stars of the W3 and W4 H II regions (Ishida 1969, 1970; Ogura and Ishida 1976) and the apparent interaction of the W3 molecular cloud with the W4 $\mathrm{H}$ II region (Lada et al. 1978). A velocity difference of about $14 \mathrm{~km} \mathrm{~s}^{-1}$ was noted between the stars in Ocl 352, which excite W4, and the gas along the W3-W4 border. A possible geometry of the W4 $\mathrm{H}$ II region and the W3 molecular cloud is displayed in Figure $3 a$ with lines of sight to various features labeled along the bottom axis (the observer is viewing these from the bottom. Consider the angle $\phi$ between the line of sight to the current center of Ocl 352 and the line joining this position with the center of the W3 molecular cloud. Unless the clouds are very elongated along our line of sight this angle must be greater than $40^{\circ}$ for the $\mathrm{H}$ II region and molecular cloud to be in physical contact. To explain the velocity difference of $\sim 14 \mathrm{~km} \mathrm{~s}^{-1}$ by expansion of the $\mathrm{H}$ II region (with an expansion velocity less than $30 \mathrm{~km} \mathrm{~s}^{-1}$ ), $\phi$ must be less than $60^{\circ}$. If W3 is to the rear of $\mathrm{W} 4$, then $\phi$ must be between $120^{\circ}$ and $140^{\circ}$, but to match a geometry with W3 in back, W4 must still be collapsing, which is unlikely in view of the excitation by so many well established main-sequence stars. Thus an angle $\phi$ of $\sim 50^{\circ}$ was adopted in Figure $3 a$. The 
location of the individual features along the line of sight is discussed below in $\S \S \mathrm{II} a(\mathrm{iv})$ and II $b(\mathrm{i})$.

\section{iii) Properties of the Star Cluster}

The open cluster Ocl 352 contains nine $O$ stars and seventy B stars (Ishida 1969). The age of Ocl 352 is estimated from its brightest main-sequence star (VSA 192 , an O5 star) to be $2.8 \times 10^{6}$ years (Ishida 1969). The age determined from a comparison of the observed $\mathrm{H}-\mathrm{R}$ diagram with theoretical isochrons ranges from $\leq 1 \times 10^{6}$ (Ishida 1969; Palouš et al. 1977) to $\sim 6$ $\times 10^{6}$ years (Stothers 1972). A more accurate determination of the age awaits observations of fainter members of the cluster because the turn-off point at ( $B$ $-V)_{0} \sim-0.2$ (corresponding to $1 \times 10^{6}$ years) is at just about the observational limit (Ishida 1969; Palouš et al. 1977).

The average radial velocity (referred to the local standard of rest) of the stars in Ocl 352 is -34 $\pm 1.4 \mathrm{~km} \mathrm{~s}^{-1}$ (Hagen 1970). However, there is a large spread in the measurements that make up this average $\left(-80\right.$ to $+84 \mathrm{~km} \mathrm{~s}^{-1}$ in the extreme; Underhill 1967). This spread is probably due to a combination of an intrinsic velocity dispersion around the mean and to a large proportion of binaries or suspected binaries in the observed sample (see Underhill 1967); it is evident from observer to observer, from star to star, and among the observations of a given star (compare Hayford 1932; Sanford 1949; Underhill 1967; Ishida 1970 and Humphreys 1976).

In the following discussions, an age of $\sim 3 \times 10^{6}$ years and a velocity of $-34 \mathrm{~km} \mathrm{~s}^{-1}$ have been adopted as most representative of the observed values of Ocl 352 , but a variance of at least $\pm 2 \times 10^{6}$ years and of $\pm 4 \mathrm{~km} \mathrm{~s}^{-1}$ is permitted by the observational uncertainties.

iv) Possible Explanations for the Observed Velocities

Galactic spiral shock.-The orientation and radial velocities of Ocl 352 and the W3 cloud shown in Figure $3 a$ are consistent with the idea that the formation of the stars was initiated at the leading edge of the original W3 cloud after its entry into the Perseus spiral arm (see Woodward 1978). The radial velocity of an object located in the Perseus arm, $2 \mathrm{kpc}$ from the Sun at $l$ $=134^{\circ}$, is $\sim-32 \mathrm{~km} \mathrm{~s}^{-1}$ because of differential galactic rotation (Schmidt 1965), but material which is just passing through the Perseus spiral shock is expected to have radial velocities between $-43 \mathrm{~km} \mathrm{~s}^{-1}$ and $-50 \mathrm{~km} \mathrm{~s}^{-1}$ (for the range of likely shock parameters given by Roberts 1972). Between 5 and $50 \times 10^{6}$ years after an object has passed through the shock, its radial velocity will become more positive with values between $-35 \mathrm{~km} \mathrm{~s}^{-1}$ and $-38 \mathrm{~km} \mathrm{~s}^{-1}$ (Roberts 1972). According to Woodward (1978), his results, which were for the passage of a cloud of mass $524 M_{\odot}$ through a galactic spiral shock, can be scaled to different cloud masses by multiplying all lengths and times by a common factor of $[M(\text { new }) / 524]^{1 / 3}$ up to about $2 \times 10^{4} M_{\odot}$ (and radius $\sim 50 \mathrm{pc}$ ), where the gravitational energy becomes comparable to the thermal energy. This mass is that estimated for the minimum contained within the three dense components of the present W3 molecular cloud, although as we say in $\S \mathrm{II} a$, the uncertain contribution of the low-density gas probably raises the total mass to near $5 \times 10^{4} M_{\odot}$. This massive object appears to have a large enough extent so that the differential pull of the galaxy across it can inhibit collapse. The detailed calculations of Woodward $(1976,1978)$ show that the passage of a cloud through the galactic spiral shock will compress and flatten the cloud at the leading edge and eventually elongate it in the opposite direction. The streaming of gas out the back of the cloud into the low-pressure medium gives rise to a velocity gradient along the length of the cloud.

The cloud begins to fragment about $4 \times 10^{7}$ years after passing through the spiral shock and the individual condensations start collapsing to form stars, such as those in Ocl 352. During the $3 \times 10^{6}$ year lifetime of Ocl 352, this cluster of stars would have moved about $30 \mathrm{pc}$ as shown in Figure $3 a$. The observed radial velocity of $-34 \mathrm{~km} \mathrm{~s}^{-1}$ for Ocl 352 is within the range expected, although Woodward's calculations predict a somewhat smaller velocity gradient downstream than the $\sim 6 \mathrm{~km} \mathrm{~s}^{-1}$ or so difference observed here; a better agreement between the observations and theory would be possible if the radial velocity of Ocl 352 were closer to $-38 \mathrm{~km} \mathrm{~s}^{-1}$, the age of Ocl 352 were at least $6 \times 10^{6}$ years, and the angle $\phi$ were $40^{\circ}$, all of which are within the observational uncertainties.

The expanding $\mathrm{H}$ il region W4.-The difference in velocity between the open cluster and the molecular gas farther downstream (including the W3 core and most of the diffuse gas) has been identified with the velocity gradient produced after passage of the initial cloud through a galactic shock. There still remains to be explained the even more negative radial velocities (around $-47 \mathrm{~km} \mathrm{~s}^{-1}$ ) observed from the zone of enhanced molecular emission along the border with the $\mathrm{W} 4 \mathrm{H}$ II region. These motions are similar to those observed in the ionized gas (Courtès 1960; Georgelin and Georgelin 1976); this suggests that a shock front is preceding the expansion of the $\mathrm{W} 4 \mathrm{H}$ II region into the neutral gas and is compressing the material into a dense layer. The expansion velocity derived from the data is $17 \pm 5 \mathrm{~km} \mathrm{~s}^{-1}$ (the uncertainty is due mainly to the leeway in adopting a stellar velocity). If one assumes that the two $\mathrm{O} 5$ stars in $\mathrm{Ocl} 352$ are ionizing the $\mathrm{W} 4 \mathrm{H}$ II region, then the current radius $s$ and electron density $n$ of the Strömgren sphere can be solved from the equations: $U=n^{2 / 3} s$ and $\mathrm{EM}=n^{2} 2 s$, where $U$ is the excitation parameter, $\sim 150 \mathrm{pc} \mathrm{cm}^{-2}$ for the two O5 stars (Panagia 1973), and EM is the emission measure, $\sim 4200 \pm 1000 \mathrm{pc} \mathrm{cm}^{-6}$ from the radio data (e.g., Wendker and Altenhoff 1977). One obtains a radius of $\sim 40 \mathrm{pc}$ and an average density of $\sim 7 \mathrm{~cm}^{-3}$. In Figure $3 a$, the shock front has been 

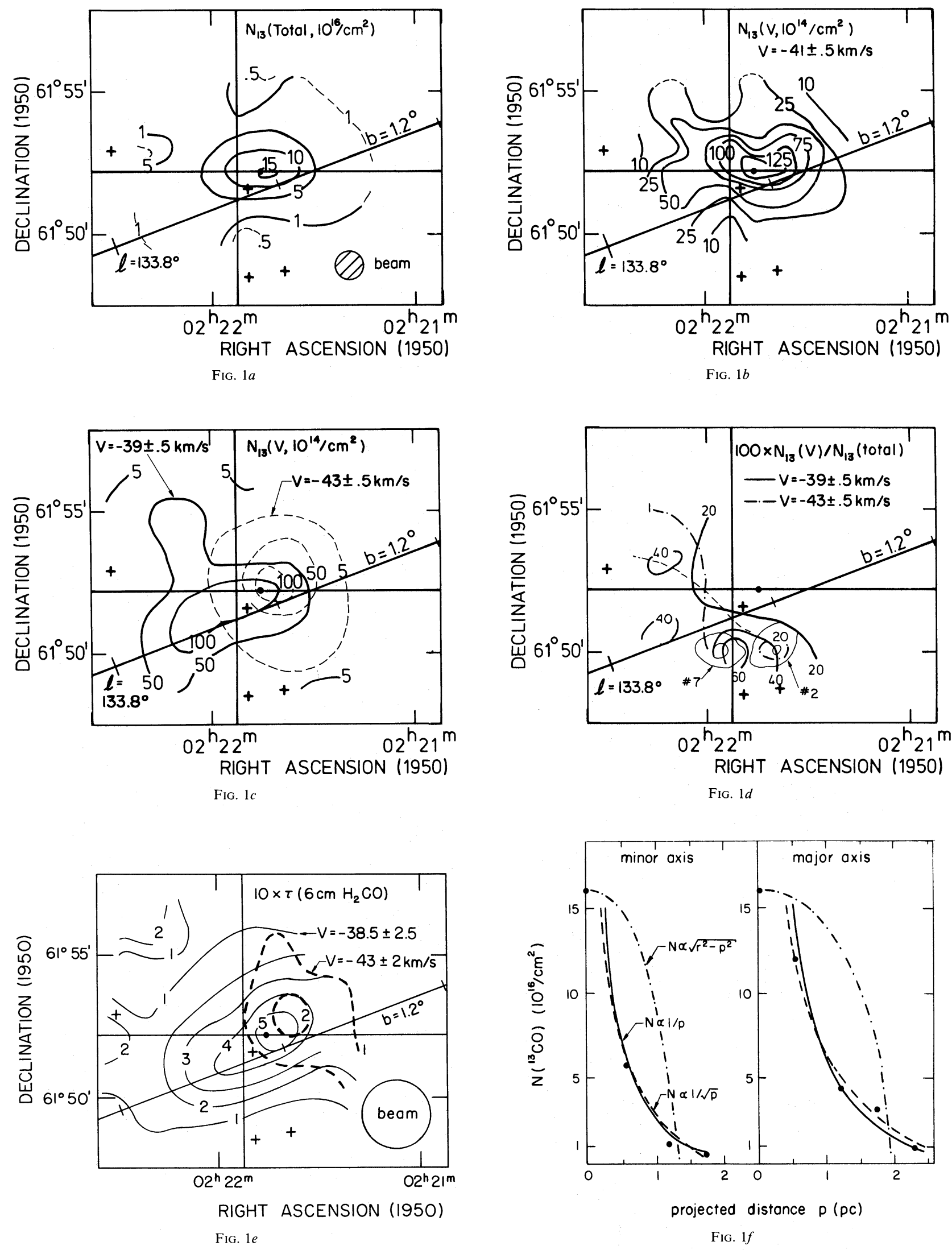
placed about $40 \mathrm{pc}$ from the current center of Ocl 352 . With this radius and expansion velocity of the $\mathrm{W} 4 \mathrm{H}$ II region plus the known age of the cluster, it is also possible to determine the initial density $n_{0}$ of the (uniform) medium into which the ionization front expanded and the current thickness of the shocked layer $\Delta s$ (see equations in Spitzer 1968). With the observed range for these parameters, $n_{0}$ could have been in the range $10-300 \mathrm{~cm}^{-3}$. With sufficient time, $\Delta s$ approaches $s / 6$. From the above range of parameters $2.6 \leq \Delta s \leq 6 \mathrm{pc}$.

The observed velocities of W3(OH), AFGL 333, and possible $\mathrm{G} 133.8+1.4$ point to their formation behind the W4 shock front. These sources are sites of recent star formation, which is probably occurring in the dense layer between the shock front and the ionization front (Elmegreen and Lada 1977; Lada, Elmegreen, and Blitz 1979). However, more detailed observational analysis (such as was done for the W3 core in $\S$ II $b$ [iii]), and further theoretical calculations are needed to confirm that the radiation pressure or stellar winds from the OB cluster can pile up enough material between the shock front and the ionization front to trigger the gravitational instabilities at the observed velocities. The two-dimensional calculations of the gas dynamics of $\mathrm{H}$ II regions by Bodenheimer, TenorioTagle, and Yorke (1979) suggest that this may be difficult because of the presence of streaming motions. A computer code to handle such calculations, including the narrow zone between the shock and ionization fronts, is currently being developed by Woodward (private communication).

An old, background $\mathrm{H}$ II region.-The observed difference in radial velocity between the W3 dense core (at $\sim-40.5 \mathrm{~km} \mathrm{~s}^{-1}$ ) and the less-dense surrounding molecular gas (at $\sim-42-44 \mathrm{~km} \mathrm{~s}^{-1}$ ) may be explained, in part, by the expansion of a large, old $\mathrm{H}$ II region located behind the northeastern quadrant of the W3 molecular cloud. The existence of this background H II region is postulated by Harten (1980) to explain distributed, low-level continuum emission seen in the radio maps of the $\mathrm{W} 3-\mathrm{W} 4$ area with the Bonn $100 \mathrm{~m}$ radio telescope. The molecular gas surrounding the northern, compact $\mathrm{H}$ II region, G133.8+1.4 (at $-44 \mathrm{~km} \mathrm{~s}^{-1}$ ) may also be under its influence. Because the exact location and extent of this old $\mathrm{H}$ II region are uncertain, the region has not been sketched in Figure $3 a$, but one can envisage the whole northeastern section of the W3 molecular cloud being caught in a squeeze

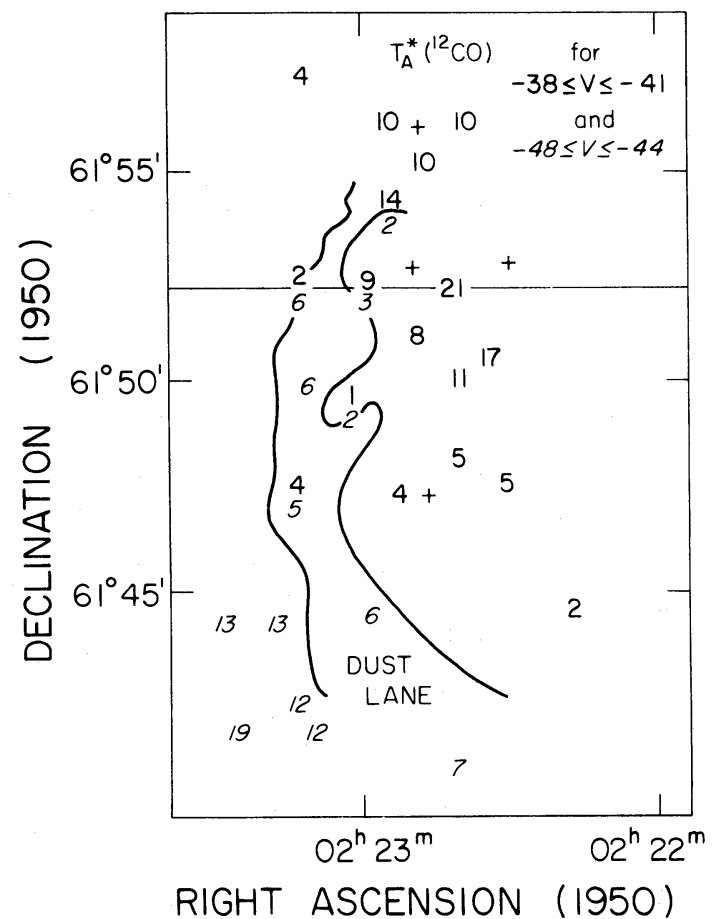

FIG. 2.-The change in intensity of the $\mathrm{CO}$ emission at two separate velocities across the (outlined) dust lane which separates the $\mathrm{H}$ II region W4 = IC 1805 (to the left) from the $\mathrm{H}$ II region W3 = IC 1795 (to the right). The $\mathrm{CO}$ antenna temperatures $T_{A}{ }^{*}$ for radial velocities between -38 and $-41 \mathrm{~km} \mathrm{~s}^{-1}$ are marked by vertical numbers and those for velocities between -44 and $-48 \mathrm{~km} \mathrm{~s}^{-1}$ by slanted numbers.

between the expansion of the $W 4 \mathrm{H}$ II region from one side and the expansion of the old $\mathrm{H}$ il region from behind.

\section{b) The Main Part of the W3 Molecular Cloud}

i) Location of the the Core Relative to the Optically-Visible $\mathrm{H}$ it Regions

The strongest emission from dust, $\mathrm{CO}, \mathrm{CS}$ and $\mathrm{HCN}$ comes from the main core of $\mathrm{W} 3$ which covers the northern group of embedded, compact $\mathrm{H}$ II regions and masers (see Fig. 3 and Table 4 in $\mathrm{D}^{2} \mathrm{WW}$ ). The average molecular velocity of the core is $\sim-40.5$ $\mathrm{km} \mathrm{s}^{-1}$, but along the northwestern edge of the diffuse optical $\mathrm{H}$ II region IC 1895 , the radial velocity of the molecular gas is $\sim-38 \mathrm{~km} \mathrm{~s}^{-1}$. The visual extinction in the area ranges from 2 to 3 mag in the

FIG. 1.-The amount of gas at various velocities. The column densities of ${ }^{13} \mathrm{CO}$ are derived under the assumption of local thermodynamic equilibrium. Note that the contour units are not the same on each map. The line of constant galactic latitude, $b= \pm 1.2^{\circ}$, is drawn. The crosses are positions of reference stars for possible alignment with optical photographs. The heavy dot shows the location of the $1720 \mathrm{OH}$ maser. $(a)$ Total ${ }^{13} \mathrm{CO}$ column density toward W3. $(b){ }^{13} \mathrm{CO}$ column density of the gas with radial velocity $V=-41 \pm 0.5 \mathrm{~km} \mathrm{~s}^{-1}$. (c) Dashed contours are ${ }^{13} \mathrm{CO}$ column densities of gas with radial velocity $V=-43 \pm 0.5 \mathrm{~km} \mathrm{~s}^{-1}$, and solid contours are for $V=-39 \pm 0.5 \mathrm{~km} \mathrm{~s}^{-1}$. The maximum (with $N$ $=120$ ) for both velocities is toward the $1720 \mathrm{MHz} \mathrm{OH}$ maser marked by the heavy dot. (d) Percentage of gas which is moving with radial velocity $V=-43+0.5 \mathrm{~km} \mathrm{~s}^{-1}$ as dot-dashed contours and with $V=-39+0.5 \mathrm{~km} \mathrm{~s}^{-1}$ as heavy solid contours. The light solid contours outline compact $\mathrm{H}$ II regions 7 (east) and 2 (west). The thin dashed line indicates the edge of the optically visible diffuse $\mathrm{H}$ II region IC 1795 , which is seen to the southeast. (e) Contours of 10 times the optical depth of $\mathrm{H}_{2} \mathrm{CO}$ at $6 \mathrm{~cm}$. Dashed curves, $V=-43 \pm 2 \mathrm{~km} \mathrm{~s}^{-1} ;$ solid curves, $V=-38$ $+2.5 \mathrm{~km} \mathrm{~s}^{-1}$. These data were obtained with the $100 \mathrm{~m}$ telescope of the Max Planck Institute of Radio Astronomy (courtesy of T. Wilson). $(f)$ Total ${ }^{13} \mathrm{CO}$ column density as a function of projected distance $p$ from the center of the W3 core along the minor axis (left) and major axis (right). 


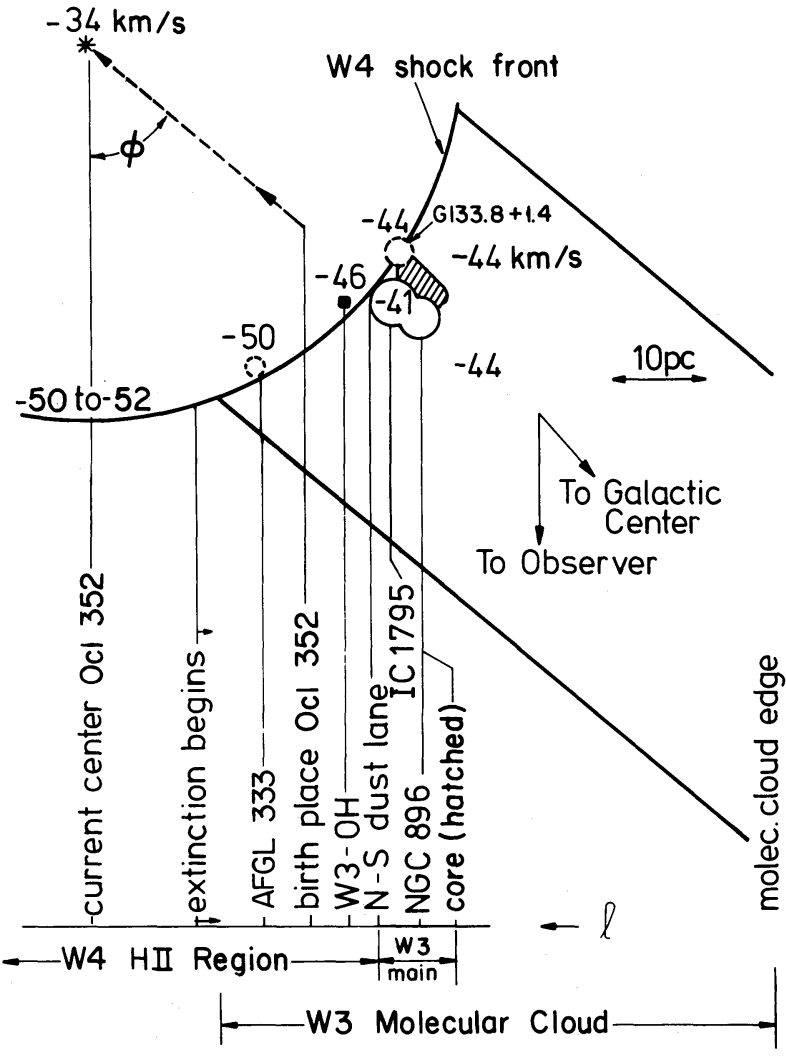

FIG. $3 a$

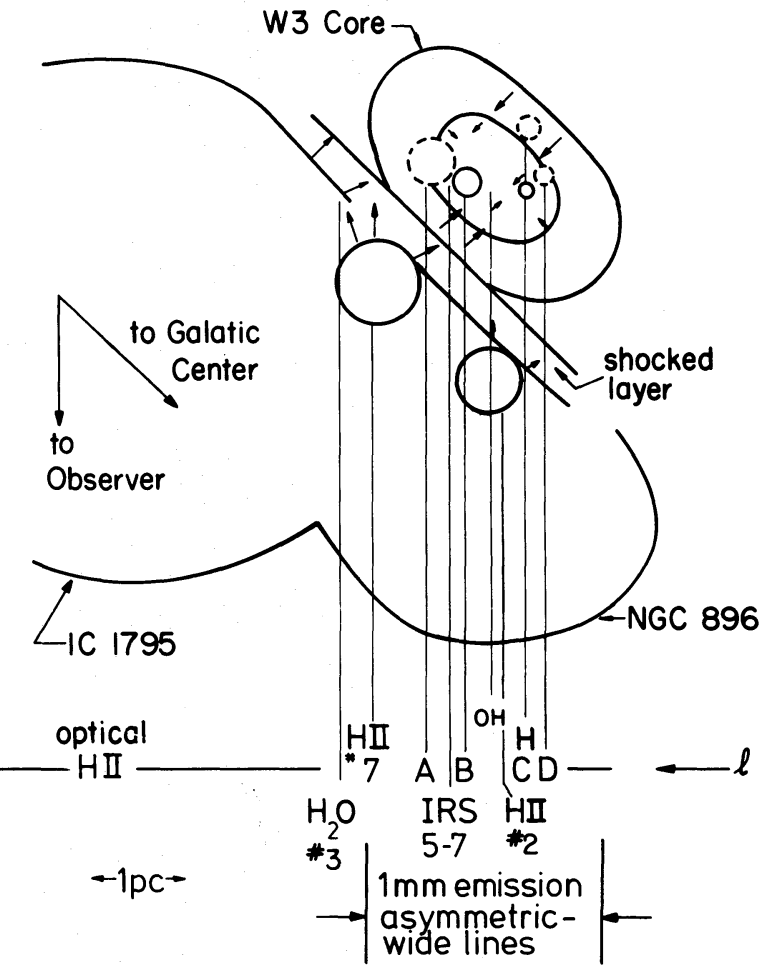

FIG. $3 b$

Fig. 3.- (a) Model of the W3-W4 region which the observer views from the bottom. Labeled lines of sight are drawn up to the various features which have been projected onto a common plane. To the left is located the W4H II region with its expanding shock front which encompasses the sources AFGL 333, W3(OH), and possibly G133.8+1.4. The positions of the cluster Ocl 352 which excites W4 are shown at birth and at the present time. Observed radial velocities are indicated at various locations. To the right is the overall $\mathrm{W} 3$ molecular cloud with the optically visible H II regions IC 1795 and NGC 896. Located behind these is the molecular core. The region W3 main is expanded in the next figure. (b) Model of the W3 main region. A cut has been made through the cloud at $b=+1.2^{\circ}$ and passes through the center of the W3 core in the direction of the (1720) $\mathrm{OH}$ maser designated $\mathrm{OH}$. The rest of the objects are projected onto this plane. The dashed circles indicate objects located above the plane (i.e., embedded $\mathrm{H}$ II regions $\mathrm{A}, \mathrm{D}$, and $\mathrm{H}$ ). The major components of the $\mathrm{W} 3$ main region are labeled along the axis at the bottom with lines of sight drawn to them. The arrows indicate motions up to about $3 \mathrm{~km} \mathrm{~s}^{-1}$.

direction of IC 1795 (Dickel and Harten 1980) to possibly 100 mag boward IRS 5 (Westbrook et al. 1976).

The W3 core, as defined by the ${ }^{13} \mathrm{CO}$ column densities in Figure $1 a$, lies to the west and north of IC 1795 and NGC 896, but some of the CO emission overlaps the region of optical emission, so there is a question of whether the molecular core is located in front of or behind the optical $\mathrm{H}$ in regions along the line of sight. The ${ }^{13} \mathrm{CO}$ column density of the core for directions near the edge of IC 1795 is at least 3 $\times 10^{16} \mathrm{~cm}^{-2}$, which implies a visual extinction in excess of 12 mag if Dickman's (1978) conversion factor applies. However, the observed extinction is about $4 \mathrm{mag}$ (from a comparison of $50 \mathrm{~cm}$ continuum emission and $\mathrm{H} \alpha$ emission by Dickel and Harten 1980). Thus the W3 core must be mostly to the north of and behind the optical $\mathrm{H}$ II regions with only a small amount overlapping in front (see Fig. 3a). A natural explanation for the more positive $\mathrm{CO}$ velocities seen along the border of the optical emission then follows, namely, the expansion of IC 1795 into the adjacent part of the molecular cloud.

The overall complex W3 main comprises the W3 core with the northern group of embedded sources, the sourthern group of compact sources, and the optically visible nebulae IC 1795 and NGC 896 (see Fig. $3 a$ and $3 b$; refer to Table 4 in $\mathrm{D}^{2} \mathrm{WW}$ ). The $\mathrm{W} 3$ main has not been placed behind the $\mathrm{W} 4$ shock front because it has a different velocity $\left(\sim-40.5 \mathrm{~km} \mathrm{~s}^{-1}\right)$ and does not appear to be participating in the expansion of W4. This can be seen in Figure 2, where the observed $\mathrm{CO}$ antenna temperatures $\left(T_{A}{ }^{*}\right)$ have been plotted for the gas with radial velocities between -38 and $-41 \mathrm{~km} \mathrm{~s}^{-1}$ (vertical numbers) and the gas with radial velocities more negative than $-44 \mathrm{~km} \mathrm{~s}^{-1}$ (slanted numbers). Both velocity components are found along the dust lane which separates the optical emission of W3 (IC 1795) to the west from that of W4 (IC 1805) to the east. Looking westward away from the dust lane 
toward $\mathrm{W} 3$, one sees that the $\mathrm{CO}$ emission at the more negative velocities weakens and disappears, while the $\mathrm{CO}$ emission at the more positive velocities becomes stronger. Conversely, one sees the gas at the more positive velocities disappearing from west to east across the dust lane. Both the optical and radio emission from IC 1795 drop abruptly at this dust lane, which also suggests a physical boundary. Therefore, we have located the W3 main at the leading edge of the W3 molecular cloud near, but not behind, the W4 shock front as shown in Figure $3 a$. The dust lane probably continues on behind IC 1795 .

An alternative possibility was presented by Lada $e t$ al. (1978), who included the W3 core behind the shock front; however, no explanation was given for its deviant velocity. Their model implies either that the shock front is moving at least $6 \mathrm{~km} \mathrm{~s}^{-1}$ slower than the ionization front (so there is a danger of the ionization front overtaking the shock front) or that there are very complicated dynamics occurring in the shocked layer.

\section{ii) Physical Conditions in the W3 Core}

Radiative transfer calculations.-The initial step in modeling the W3 molecular core was to assume a homogeneous, spherical cloud and search for sets of parameters which could simultaneously reproduce the observed peak intensities of the $J=1-0$ spectral lines of ${ }^{12} \mathrm{CO}$ and ${ }^{13} \mathrm{CO}$, the $J=2-1$ line of $\mathrm{CS}$, and the $J$ $=1-0, F=2-1$ line of $\mathrm{HCN}$ in the center of the cloud (i.e., toward embedded $\mathrm{H}$ II region $\mathrm{H}$ and the 1720 $\mathrm{MHz} \mathrm{OH}$ maser designated $\mathrm{OH}$ in Fig. $3 b$ ). In addition, the model calculation also gave the optical depth in each line which was compared with some observational constraints. The radiative transfer was calculated using a multilevel Sobolev approximation adapted from the work of de Jong, Chu, and Dalgarno (1975) (see also Goldreich and Kwan 1974; Scoville and Solomon 1974). The collision cross sections for $\mathrm{CO}$ and CS were taken from the paper by Green and Chapman (1978) and for HCN from the paper by Green and Thaddeus (1974). These models assumed a velocity gradient through the cloud arising from contraction. If the dimensions and kinetic temperature of the cloud plus the general magnitude of the velocity field are chosen to agree with the observed ones, then similar solutions to the radiative transfer problems are obtained for either a static microturbulent or a collapsing cloud model (White 1977). This was confirmed by comparing results of the calculations in this work, using the Sobolev approximation, with those of Lucas (1976), Liszt and Leung (1977) and Leung (1978), who considered turbulence-broadened lines without the Sobolev approximation. For the same model clouds and an equivalent velocity width, there were no substantial deviations between the maximum line intensities or central optical depths predicted by either method. To distinguish between a velocity gradient and turbulence components, a more sophisticated analysis and comparison of predicted line shapes with the observed ones is required (e.g., Lucas 1976; Leung 1978; and Kwan 1978). Some comparisons of the observed line shapes with theoretical ones will be made after the presentation of the basic parameters of the cloud below.

Input parameters. - The physical parameters which were found from the observations $\left(\mathrm{D}^{2} \mathrm{WW}\right)$ and the range used in the calculations are listed in Table 1 . The appropriate velocity gradient $d V / d r=V / R$ to use in the Sobolev approximation was obtained from the observed line widths and radius. The maximum observed ${ }^{13} \mathrm{CO}$ line width measured between the halfintensity points, $\Delta V$, is around $7 \mathrm{~km} \mathrm{~s}^{-1}$, so that the appropriate $V$ (which is $\frac{1}{2} \Delta V$ ) is $3.5 \mathrm{~km} \mathrm{~s}^{-1}$. The depth through the core must be larger than the diameter of the largest embedded $\mathrm{H}$ in region $\mathrm{A}(\sim 0.5 \mathrm{pc})$ and is probably no larger than the major axis $(\sim 4 \mathrm{pc})$, and so I have taken it as equal to the length of the minor axis ( $\sim 2.5 \mathrm{pc}$ ), or $R=1.2 \mathrm{pc}$.

Results for a uniform cloud.-The observations to be matched by the model are listed in the upper panel A of Table 2. The values of the optical depths were determined by taking into account the observed effects of self-absorption in the ${ }^{12} \mathrm{CO}$ profile and to a lesser degree in the CS profile. For this effect to be evident, their optical depths $\tau$ must be $\gtrsim 3$, and because the effect is less pronounced in CS, probably $\tau(\mathrm{CS}) \lesssim \tau\left({ }^{12} \mathrm{CO}\right)$. The picture is more complicated for $\mathrm{HCN}$ because of the hyperfine structure, but an optical depth of $\sim 10$ is reasonable from the analysis of Gottlieb et al. (1975). The weakness of the CS and $\mathrm{HCN}$ lines relative to the ${ }^{12} \mathrm{CO}$ line is because $\mathrm{CS}$ and $\mathrm{HCN}$ require optical depths of at least 10 and 100 , respectively, before a line becomes saturated, that is,

TABLE 1

InPut Parameters for Radiative Transfer Calculations

\begin{tabular}{|c|c|c|}
\hline Parameters & Specified by the Data & Range Used \\
\hline 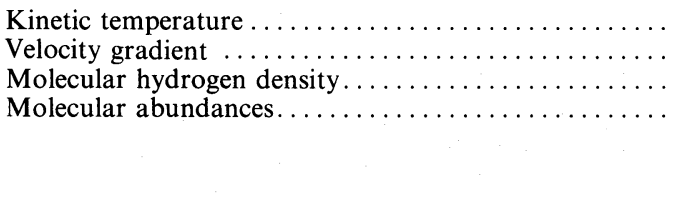 & $\begin{array}{c}35 \leq T_{\mathrm{K}} \leq 100 \mathrm{~K} \\
d v / d r \sim 3 \mathrm{~km} \mathrm{~s}^{-1} \mathrm{pc}^{-1} \\
\cdots \\
\cdots\end{array}$ & $\begin{array}{l}T_{K}=10-100 \mathrm{~K} \\
d v / d r=1-15 \mathrm{~km} \mathrm{~s}^{-1} \mathrm{pc}^{-1} \\
n_{\mathrm{H}_{2}}=10^{2}-10^{6} \mathrm{~cm}^{-3} \\
\left.{ }^{12} \mathrm{CO}\right] /\left[\mathrm{H}_{2}\right]=10^{-5}-10^{-4} \\
{\left[{ }^{12} \mathrm{CO}\right] /\left[{ }^{13} \mathrm{CO}\right]=20-90} \\
\left.[\mathrm{CS}] / \mathrm{H}_{2}\right]=10^{-11}-10^{-8} \\
{[\mathrm{HCN}] /\left[\mathrm{H}_{2}\right]=10^{-11}-10^{-8}}\end{array}$ \\
\hline
\end{tabular}


TABLE 2

Results of Radiative Transfer Calculations

\begin{tabular}{|c|c|c|c|c|}
\hline \multicolumn{5}{|c|}{ A. Observations to be Reproduced } \\
\hline & $\mathrm{CS}$ & ${ }^{12} \mathrm{CO}$ & ${ }^{13} \mathrm{CO}$ & $\mathrm{HCN}$ \\
\hline $\begin{array}{l}T_{A}{ }^{*}(\mathrm{~K}) \\
\tau^{\mathrm{a}} \ldots \ldots \ldots\end{array}$ & $\begin{array}{c}2-2.5 \\
\gtrsim 3\end{array}$ & $\begin{array}{c}30-31 \\
\gtrsim 3\end{array}$ & $\begin{array}{l}5-6 \\
\lesssim 1\end{array}$ & $\begin{array}{c}2.5 \\
(\lesssim 10 ?)\end{array}$ \\
\hline
\end{tabular}

B. UNIForm Cloud Solutions

\begin{tabular}{|c|c|c|}
\hline Parameters & Case 1 (all data) & Case 2 (without $\mathrm{HCN}$ ) \\
\hline $\begin{array}{l}\text { Velocity gradient } \ldots \ldots \ldots \ldots \ldots \ldots \ldots \ldots \ldots \ldots \ldots \ldots \\
\text { Kinetic temperature } \ldots \ldots \ldots \ldots \ldots \ldots \ldots \ldots \ldots \ldots \ldots \\
\text { Molecular hydrogen density } \ldots \ldots \ldots \ldots \ldots \ldots \ldots \ldots \\
\text { Relative abundances and associated optical depths }\end{array}$ & $\begin{array}{l}d v / d r \sim 3 \mathrm{~km} \mathrm{~s}^{-1} \mathrm{pc}^{-1} \\
T_{K} \sim 35-40 \mathrm{~K} \\
n_{\mathrm{H} 2} \sim(1-2) \times 10^{4} \mathrm{~cm}^{-3} \\
{[\mathrm{CS}] /\left[\mathrm{H}_{2}\right] \sim(2-5) \times 10^{-9}, \tau(\mathrm{CS}) \sim 3-4} \\
{[\mathrm{HCN}] /\left[\mathrm{H}_{2}\right] \sim(3-8) \times 10^{-9}, \tau(\mathrm{HCN}) \sim 10-15} \\
{\left[{ }^{12} \mathrm{CO}\right] /\left[\mathrm{H}_{2}\right] \gtrsim 3 \times 10^{-5}, \tau\left({ }^{12} \mathrm{CO}\right) \gtrsim 5} \\
{\left[{ }^{12} \mathrm{CO}\right] /\left[{ }^{13} \mathrm{CO}\right] \gtrsim 40, \tau\left({ }^{13} \mathrm{CO}\right) \lesssim 0.5}\end{array}$ & $\begin{array}{l}d v / d r \sim 3 \mathrm{~km} \mathrm{~s}^{-1} \mathrm{pc}^{-1} \\
T_{K} \sim 35 \mathrm{~K} \\
n_{\mathrm{H}_{2}} \sim 5 \times 10^{3} \mathrm{~cm}^{-3} \\
{[\mathrm{CS}] /\left[\mathrm{H}_{2}\right] \sim 2 \times 10^{-8}, \tau(\mathrm{CS}) \sim 7} \\
\left.{ }^{12} \mathrm{CO}\right] /\left[\mathrm{H}_{2}\right] \sim 10^{-4}, \tau\left({ }^{12} \mathrm{CO}\right) \sim 10 \\
{\left[{ }^{12} \mathrm{CO}\right] /\left[{ }^{13} \mathrm{CO}\right] \sim 89, \tau\left({ }^{13} \mathrm{CO}\right) \lesssim 0.5}\end{array}$ \\
\hline
\end{tabular}

${ }^{\mathrm{a}} \tau(\mathrm{CS}) \lesssim \tau\left({ }^{12} \mathrm{CO}\right)$.

before $T_{A}{ }^{*}$ approaches $T_{K}$. For $\mathrm{CO}$ this happens at much lower optical depths because of its small dipole moment (Leung 1978).

The results of the radiative transfer calculations are given in the lower panel B of Table 2. The values listed under case 1 constitute our best set of parameters for a uniform cloud model which simultaneously reproduces the observed line intensities. If the molecular hydrogen densities are much higher than listed there, then to produce the same CS intensity, the CS density must be reduced, which causes its optical depth to become too low. If the $\mathrm{H}_{2}$ densities are much lower, then the HCN optical depth must become enormous, requiring selfabsorption effects larger than observed. If the kinetic temperature is above $40 \mathrm{~K}$, the $\mathrm{CO}$ observations become increasingly more difficult to fit because then $\tau\left({ }^{12} \mathrm{CO}\right)<3$ and $\tau\left({ }^{12} \mathrm{CO}\right)<\tau(\mathrm{CS})$; furthermore, for $T_{K}>40 \mathrm{~K}$ one has $\left[{ }^{12} \mathrm{CO}\right] /\left[\mathrm{H}_{2}\right]<3 \times 10^{-5}$ and $\left[{ }^{12} \mathrm{CO}\right] /\left[{ }^{13} \mathrm{CO}\right]<30$.

Unless higher optical depths can be tolerated for $\mathrm{HCN}$ or excitation by electron collisions is significant, it is not possible to lower the average density below $10^{4} \mathrm{~cm}^{-3}$ and fit all the data. However, if this restriction on the density imposed by the HCN data can be relaxed, then the solution listed as case 2 in Table 2 is possible. Once again, solutions with higher temperatures or lower densities are ruled out.

It would be possible to distinguish between the cases where $\left[\mathrm{H}_{2}\right] /\left[{ }^{13} \mathrm{CO}\right]=0.9 \times 10^{6}$ and $1.3 \times 10^{6}$ if accurate measurements of the intensities of the $J=3-2$ or $J=2-1$ lines of ${ }^{13} \mathrm{CO}$ were available. The intensity of these lines is comparable to the $J=1-0$ intensity if $\left[\mathrm{H}_{2}\right] /\left[{ }^{13} \mathrm{CO}\right] \sim 0.9 \times 10^{6}$, whereas it is about twice that of the $J=1-0$ line if $\left[\mathrm{H}_{2}\right] /\left[{ }^{13} \mathrm{CO}\right] \sim 1.3 \times 10^{6}$.

The predicted line shapes for ${ }^{12} \mathrm{CO},{ }^{13} \mathrm{CO}$, and $\mathrm{CS}$ emission form a uniform cloud with the above parameters and various forms for the velocity gradient can be compared with our observed profiles to give some indication of the velocity law governing the contraction of the W 3 core. First, the quantity $\alpha$ defined by Leung (1978) as the ratio of the systematic to turbulent velocity, $v_{z} / v_{t}$, must be estimated. From our observations $\left(\mathrm{D}^{2} \mathrm{WW}\right)$ we find that $1 \leq v_{z} \leq 3$ and $1.5 \leq v_{t}$ $\leq 3 \mathrm{~km} \mathrm{~s}^{-1}$ : thus $0.3 \leq \alpha \leq 2$ for the W3 core. The agreement between the calculated profiles in Figure $5 a$ of the paper by Leung (1978) and the observed ones, especially toward $\mathrm{H}$ and IRS 5-6-7 (Fig. $5 a$ in $\mathrm{D}^{2} \mathrm{WW}$ ) is very good for $v=$ (constant) or $v \propto r$; however, $v \propto(1 / r)^{1 / 2}$ can be ruled out.

The solutions given in Table 2, though not unique, bound the range of physical parameters allowed for a uniform cloud.

Density gradients.--The ${ }^{13} \mathrm{CO}$ column densities as a function of projected distance $p$ from the center of the W3 core along the minor axis and major axis are plotted in Figure $1 f$. The rate of decrease is faster than expected for a cloud of uniform density (which is shown by the dot-dashed line). Outside the very center, the column density decreases inversely with the projected distance (solid line) or possibly the square root of the projected distance (dashed line), which means that $n_{\mathrm{H}_{2}} \propto\left(r^{-2}\right.$ or $\left.r^{-1.5}\right)$. Inside, however, even after allowing for resolution effects over distances smaller than a beamwidth $(\sim 0.6 \mathrm{pc})$, one still finds that the density gradient must flatten toward the center. With an abundance ratio $\left[\mathrm{H}_{2}\right] /\left[{ }^{13} \mathrm{CO}\right]$ in the range $(0.9-$ $1.3) \times 10^{6}$ as found in $\S$ II $b[$ iii], the average density of molecular hydrogen in the inner region is $\sim 5$ $\times 10^{4} \mathrm{~cm}^{-3}$. This falls below $\sim 5 \times 10^{3} \mathrm{~cm}^{-3}$ in the outer parts beyond the $N\left({ }^{13} \mathrm{CO}\right) \sim 10^{16} \mathrm{~cm}^{-2}$ contour in Figure $1 a$. These results are in agreement with the hydrogen densities determined from the analysis of the $1 \mathrm{~mm}$ emission from dust by Westbrook et al. (1976). The ${ }^{13} \mathrm{CO}$ intensity becomes too weak to follow the density contours past the $N\left({ }^{13} \mathrm{CO}\right) \sim 10^{16} \mathrm{~cm}^{-2}$ level, but if the molecular density continues the same 
decrease out to twice the measured size, then the amount of mass enclosed by $N=10^{16} \mathrm{~cm}^{-2}$ is about $80 \%$ of the total mass in this condensation.

Visual extinction.-Dickman (1978) has found that $A_{V}(\mathrm{mag})=(4.0 \pm 2.0) \times 10^{-16} N_{13}(\mathrm{LTE})$ for dark clouds over a range $1.5 \leq A_{V}(\mathrm{mag}) \leq 10$. It is of interest to see whether the relationship extends to more opaque clouds such as the W3 core. This will be discussed in a later paper where the visual extinctions inferred from published $1 \mathrm{~mm}$ observations, near-IR spectra, and our radio-optical study (Dickel and Harten 1980) will be compared with the ${ }^{13} \mathrm{CO}$ column densities found here.

\section{iii) Motions in the W3 Core}

Effects of the expanding $\mathrm{H}$ II regions on adjacent parts of the molecular core.-We have previously discussed the expansion of the W4 ionization front into the overall W3 molecular cloud. Now we shall see that, within W3 itself, the nebula IC 1795 and the compact $\mathrm{H}$ in regions 7 and 2 (refer to Fig. $3 b$ and to Sullivan and Downes 1973) are expanding into the adjacent molecular core. The initial evidence for this comes from the observation that the lines of constant velocity run parallel to and are most positive along the optical border (Figs. 7 and $8 b$ in $\mathrm{D}^{2} \mathrm{WW}$ ). To investigate the interaction in more detail, I have analyzed the ${ }^{13} \mathrm{CO}$ column density in this direction as a function of velocity. The amount of gas with radial velocities within $1 \mathrm{~km} \mathrm{~s}^{-1}$ intervals centered on the velocities $-41,-39$, and $-43 \mathrm{~km} \mathrm{~s}^{-1}$ are shown in Figures $1 b$ and $1 c$. The mean velocity of the W3 core is $-41 \mathrm{~km} \mathrm{~s}^{-1}$. As expected for typical line widths of 8 $10 \mathrm{~km} \mathrm{~s}^{-1}$, the gas in each interval near the line center accounts for $10-20 \%$ of the total amount present. The distributions of the ${ }^{13} \mathrm{CO}$ column densities for velocities displaced $2 \mathrm{~km} \mathrm{~s}^{-1}$ from the mean (Fig. 1c) should be identical. Indeed, both distributions have their maxima in the direction of the $1720 \mathrm{OH}$ maser (the heavy dot) and are similar over much of the W3 core. However, while the column densities of the gas at $-43 \mathrm{~km} \mathrm{~s}^{-1}$ are symmetrical around the $\mathrm{OH}$ maser and fall off sharply toward the optical border to the east, the amount of gas at $-39 \mathrm{~km} \mathrm{~s}^{-1}$ remains very high in this direction. A similar pattern is seen for the optical depths of $\mathrm{H}_{2} \mathrm{CO}$ at the two velocities (Fig. 1e). The discrepancy is enhanced when the $\mathrm{CO}$ data are represented as the percentages of the gas at either velocity versus position. In Figure $1 d$ the percentage of the gas at $-43 \mathrm{~km} \mathrm{~s}^{-1}$ and at $-39 \mathrm{~km} \mathrm{~s}^{-1}$ is shown by the dot-dashed and the heavy solid curves, respectively. The thin dashed curve represents the edge of the optical emission, and the thin solid curves indicate the location of the partially obscured compact $H$ II regions 7 and 2 . It is seen that the gas at $-43 \mathrm{~km} \mathrm{~s}^{-1}$ amounts to less than $1 \%$ of the total over much of the region where optical emission is seen, whereas the gas at $-39 \mathrm{~km} \mathrm{~s}^{-1}$ is $20-40 \%$ of the total and reaches $60 \%$ near source 7 . There is very little molecular gas at all along the line of sight through source 7 (only $5 \%$ of the amount along the line of sight through the center of the W3 core), but what is there appears to be moving with a (radial) velocity around $-39 \mathrm{~km} \mathrm{~s}^{-1}$.

From the above analysis it is concluded that (1) the optical $\mathrm{H}$ II regions are influencing the motions of the gas in the adjacent molecular core, but (2) this is a minor effect in terms of the overall gas in the W3 core. In other words, shock fronts associated with the $\mathrm{H}$ II regions are moving into the southern edge of the molecular core, but they have by no means crossed the entire $W 3$ core and precipitated its collapse.

Can rotation stop the collapse?-It appears that the observed line widths, velocity gradient, and line shapes can be adequately explained by a combination of turbulent and collapse motions and an expansion of the $\mathrm{H}$ II regions into the edge of the molecular core. As an alternative, one should consider the importance of possible rotation and whether or not it can equally well account for the observed characteristics of the W3 core. Indeed, the plots of radial velocities in Figures 7 and $8 b$ of the previous paper $\left(\mathrm{D}^{2} \mathrm{WW}\right)$ resemble the rotation curves of many external galaxies. If one measures the radial velocity along a line in the direction of the maximum gradient, then one can estimate the total mass of the W3 system $T_{T}$ by the relation $M_{T}=(3 / 2)^{3 / n} V_{\max }{ }^{2} R_{\max } \csc ^{2} i / G$ (Brandt 1960). For the $\mathrm{W} 3$ core, the exponent $n \gtrsim 3$ and the maximum radial velocity $V_{\max }=2.75 \mathrm{~km} \mathrm{~s}^{-1}$ at a distance of $R_{\max }=0.5 \mathrm{pc}$. The total mass is $1.2 \times 10^{3} M_{\odot}$ for an edge-on system. If the inclination $i$ were $57^{\circ}$ (yielding the observed axial ratio of 0.63 for the W3 core), then the total mass would be $2 \times 10^{3} M_{\odot}$. This is still less than half the minimum mass derived in $\S$ II $a$ [i] for the W3 core [within the $N\left({ }^{13} \mathrm{CO}\right) \sim 10^{16} \mathrm{~cm}^{-2}$ contour of Fig. 1a].

Although the whole cloud cannot be held up by rotation, perhaps the centrifugal and gravitational forces balance in the inner parts. If the elliptically shaped core (enclosed by the $N=5 \times 10^{16} \mathrm{~cm}^{-2}$ contour) is an embedded Maclaurin spheroid with an ellipticity of 0.8 and a major axis of $1.2 \mathrm{pc}\left(2^{\prime}\right)$, then the mass of this equilibrium figure (according to eqs. [3227] and [3-234] of Lang 1974) would be 1.3 $\times 10^{3} M_{\odot}$, which is about half the minimum mass present in the inner region. So although rotation is not entirely ruled out and may help retard the collapse of the core, it cannot itself prevent this collapse.

There are other serious problems with the rotatingcore model. The line connecting the extreme radial velocities does not go through the center of the dense core, and it is not along the major axis of the W3 core but at a $45^{\circ}$ angle to it. The velocity gradient is also not parallel to the galactic plane (but at an angle of $\sim 20^{\circ}$ ).

It is primarily because the velocity gradient is perpendicular to the optical edge where the molecular core and IC 1795 interact that the interpretation of an expanding $\mathrm{H}$ II region is preferred. In this case, the more negative velocities of the embedded sources $C, D$, 
and $\mathrm{H}$ can be explained by location of these components in the real half of the collapsing cloud. The relatively large optical depths of $\mathrm{H}_{2} \mathrm{CO}$ toward these sources (Fig. 1e) support this conclusion. To clarify the situation further, one needs observations with higher spatial resolution as well as calculations of the transfer of radiation through model clouds which have both density gradients plus some rotation, as well as collapse and turbulence. Such calculations should perhaps apply the statistical Monte Carlo approach of Bernes (1979) so that nonspherical geometries with embedded sources could be considered. These would not give a unique answer, but might rule out certain configurations.

iv) Model of the W3 Core

Figure $3 b$ is a final sketch of the model of the W3 core based upon the results of the preceding sections. Along the line of sight, one first encounters the large diffuse optical, H II region IC 1795 (left) and the brighter but somewhat smaller H II region NGC 896 (right). These are located mostly below the plane of the drawing. The next smaller pair of circles along the line of sight are the compact $\mathrm{H}$ II regions 7 (left) and 2 (right) which are just below the plane. Immediately behind them is the compressed layer of gas due to the shock front expanding into the molecular core. Only the column density of this layer is accurately known, not its depth. For a thickness as large as $0.3 \mathrm{pc}$ (or $0.5 \mathrm{pc}$ depth along the line of sight), the average molecular hydrogen density is $\sim 2 \times 10^{3} \mathrm{~cm}^{-3}$ but increases to about $6 \times 10^{3} \mathrm{~cm}^{-3}$ just behind the compact $\mathrm{H}$ II region 7 . Since the radial velocities (= -38.7 and $-37.6 \mathrm{~km} \mathrm{~s}^{-1}$ ) of the most intense components of the $\mathrm{H}_{2} \mathrm{O}$ maser 3 located near this compact $\mathrm{H}$ II region are the same as the gas behind the shock front, I suggest that this $\mathrm{H}_{2} \mathrm{O}$ maser is located therein. In this case, the thickness of the layer must be much less than shown to get the high densities expected near the maser, or else there are significant concentrations.

The rest of the molecular core is located further along the line of sight and is represented by two elliptical contours. Within the inner contour, $\left\langle n_{\mathrm{H}_{2}}\right\rangle$ $\sim 5 \times 10^{4} \mathrm{~cm}^{-3}$; the outer contour represents $\left\langle n_{\mathrm{H}_{2}}\right\rangle$ $\sim 5 \times 10^{3} \mathrm{~cm}^{-3}$. The density falls to about $10^{2} \mathrm{~cm}^{-3}$ over the remainder of the W3 molecular cloud. The solid and dashed circles approximately locate the embedded compact $\mathrm{H}$ II regions within the W3 molecular core.

The arrows in Figure $3 b$ are velocity vectors which schematically depict the few $\mathrm{km} \mathrm{s}^{-1}$ expansion of the shock in the W3 core and the similar amount of collapse motion within the W3 core. Although the vectors drawn in the figure suggest contraction with $v \propto r$, a uniform contraction at constant velocity is within the observational errors.

The overall characteristics of the W3 core (aside from the expanding shock front and the existence of embedded condensations) are similar to those resulting from a model calculation by Gerola and Glassgold
(1978) for a cloud of $2 \times 10^{4} M_{\odot}$ with an initially uniform density of $150 \mathrm{~cm}^{-3}$. After one free-fall time $\left(\sim 3 \times 10^{6} \mathrm{yr}\right)$ the atomic hydrogen has been nearly completely converted to molecular hydrogen, and, for their "warm" case, the abundance ratio $\left[{ }^{12} \mathrm{CO}\right] /\left[\mathrm{H}_{2}\right]$ has reached $\sim 6 \times 10^{-5}$. Like the W3 core, their model has a kinetic temperature of $35 \mathrm{~K}$ and the density in the inner $1 \mathrm{pc}$ is fairly constant at $5 \times 10^{4} \mathrm{~cm}^{-3}$, falling to $10^{3} \mathrm{~cm}^{-3}$ at $2.7 \mathrm{pc}$. The collapse velocity in the inner parts is a few $\mathrm{km} \mathrm{s}^{-1}$ and reaches a maximum of 5$6 \mathrm{~km} \mathrm{~s}^{-1}$ in the outer 2-3 pc.

\section{v) Variation in the CS Abundance}

The CS emission.-All the observational features summarized in $\S$ I have been discussed except the anaomalous behavior of $\mathrm{HCN}$ and CS toward IRS 56-7. Over the rest of the core, the HCN and CS lines have comparable intensities, but toward IRS 5-6-7 the $\mathrm{HCN}(J=1-0, G=2-1)$ line remains at $T^{*} \sim 2.5 \mathrm{~K}$ and resembles the wide, self-absorbed ${ }^{12} \mathrm{CO}$ line whereas the CS $(J=2-1)$ line nearly disappears. In the following sections, several possibilities are considered for explaining this situation. They are (1) a constant relative abundance of CS throughout, accompanied by a change in the relative population of the levels; (2) a change in the relative CS abundance due to the presence of shocks in the vicinity of IRS 5; and (3) a change in the average CS abundance related to ionmolecule chemistry occurring in the different parts of the cloud. For convenience in the discussion that follows, the direction of IRS 5-6-7 will be shorted to IRS 5, and the conditions in the rest of the cloud will be exemplified by those determined for the line-of-sight toward the embedded source $\mathrm{H}$.

Possible changes in the relative population of the levels.-Perhaps the molecular hydrogen density is locally high enough near IRS 5 to populate the higher $J$ levels of CS so that the $J=3-2$ line becomes more intense than the $J=2-1$ line. Evidence for higher densities includes the facts that the ${ }^{13} \mathrm{CO}$ column density is 1.5 times that at $\mathrm{H}$, the $1 \mathrm{~mm}$ emission form dust peaks on IRS 5 (Westbrook et al. 1976), the $9.7 \mu \mathrm{m}$ absorption is strongest toward IRS 5 (Hackwell et al. 1978), and an $\mathrm{H}_{2} \mathrm{O}$ maser is present. For the kinetic temperature and relative abundance found toward $\mathrm{H}$, the intensity of the $J=2-1$ line will remain greater than that of the $J=3-2$ line until a molecular hydrogen density of at least $10^{5} \mathrm{~cm}^{-3}$ is reached. At that point, however, $T_{A}{ }^{*}$ should be $\gtrsim 10 \mathrm{~K}$ for both lines! If the temperature instead of the density is increased, then again the intensities of both lines increase. Thus increasing the excitation to higher levels by either increasing the density or temperature results in a higher, not lower, intensity for the $J=2-1$ line of $\mathrm{CS}$, not to mention the corresponding increase in the intensities of the ${ }^{13} \mathrm{CO}$ and $\mathrm{HCN}$ lines above those observed at IRS 5. Thus it is concluded that it is not possible to vary the relative populations of the levels in the way needed to explain the variation in CS intensities. 
Decrease in CS abundance toward IRS 5.-The observed decrease in the intensity of the CS emission toward IRS 5 can be accounted for by decreasing the average CS abundance by a factor of about 5 if all other parameters remain constant and equal to those at $\mathrm{H}$. Whether one invokes shock chemistry or timedependent ion-molecule chemistry within a dense, shielded region, the relative abundances of $\mathrm{H}_{2},{ }^{12} \mathrm{CO}$, ${ }^{13} \mathrm{CO}$, and $\mathrm{HCN}$ must remain essentially unchanged while the CS abundance decreases.

Before considering the predictions from shock and ion-molecule chemistry, let us first investigate IRS 5. What is so special about this region to cause a decrease in CS abundance compared to the rest of the cloud? The most obvious thing is the very existence of this young protostellar cluster IRS 5 with its associated maser emission $\left(\mathrm{H}_{2} \mathrm{O} 2\right)$ and essentially no radio continuum emission. The conditions in the direction of this object are slightly different from the mean values toward the cloud center derived above, so we present here the best values for the parameters toward IRS 5 itself. If the relative abundance of ${ }^{13} \mathrm{CO}$ is to remain constant throughout the cloud, then it is somewhat easier to fit the data toward both IRS 5 and the center of the cloud $(\mathrm{H})$ with the lower value of $0.9 \times 10^{6}$ for $\left[\mathrm{H}_{2}\right] /\left[{ }^{13} \mathrm{CO}\right]$ found in $\S \mathrm{II} b[\mathrm{ii}]$. A somewhat lower kinetic temperature $\left(T_{\mathrm{K}} \sim 30 \mathrm{~K}\right)$ and a doubling of the average molecular hydrogen density can account for the ${ }^{12} \mathrm{CO}$ and ${ }^{13} \mathrm{CO}$ intensities toward IRS 5. A less steep density gradient coupled with higher internal motions near the protostars might explain the broad, self-absorbed HCN profile at IRS 5. To obtain the same observed $\mathrm{HCN}$ intensity at double the average $\mathrm{H}_{2}$ density requires a reduction of the relative abundance of $\mathrm{HCN}$ by a factor of $\sim 4$, and to account for the fourfold drop in CS intensity under these circumstances requires a reduction in the CS abundance by a factor of 15-20.

1. Shock chemistry.-According to the calculations of Iglesias and Silk (1978) for a $10 \mathrm{~km} \mathrm{~s}^{-1}$ shock propagating into a dense molecular cloud, the abundances of $\mathrm{CO}$ and $\mathrm{HCN}$ are unperturbed by the shock as required here, but according to the calculations of Hartquist, Oppenheimer, and Dalgarno (1980), the CS abundance is expected to increase - not decrease. Thus shock chemistry appears to be ruled out for explaining the variation in CS abundance.

2. Time-dependent ion-molecule chemistry.-In addition to poorly known elemental abundances in dense clouds, several other factors can affect the results of calculations of the ion-molecule chemistry: there are uncertainties in the reaction rates, some seemingly unimportant constituent of the gas may have been excluded, the average interstellar radiation field varies from place to place, the cosmic-ray ionization rate might be lower in dense clouds, and condensation on dust may be important (e.g., Iglesias 1977). Despite the pitfalls present in trying to trace the growth of a particular molecule, I wish to point out one trend in the results from both the time-dependent calculations of
Henning (1979) and the steady-state calculations of Mitchell, Ginsburg, and Kuntz (1978). For hydrogen densities between about $10^{3}$ and $10^{4} \mathrm{~cm}^{-3}$, the curve for the relative abundance of $\mathrm{HCN}$ reaches a broader maximum before decreasing than the corresponding curve for CS. Thus, between $n \sim 10^{3} \mathrm{~cm}^{-3}$ and $n \sim 5$ $\times 10^{3} \mathrm{~cm}^{-3}$ the $\mathrm{HCN}$ abundance may decrease by only a factor of 3 while the CS abundance drops by a factor of 10-20. This is the sort of behavior inferred earlier from the observations toward IRS 5. In summary, the ion-molecule chemistry predicts that after initially increasing to a maximum, the relative abundances of both $\mathrm{HCN}$ and $\mathrm{CS}$ decrease with increasing density, and in some parts of the cloud the CS abundance may fall faster. At the moment, such a situation is the only viable explanation of the low CS intensity observed towards IRS 5.

\section{SUMMARY}

\section{a) Current Physical Parameters}

From an analysis of the molecular data I have found the following parameters to characterize the physical conditions in the core of the W3 molecular cloud: the kinetic temperature is $35 \mathrm{~K}$; the average molecular hydrogen density within $\sim 0.5 \mathrm{pc}$ of the center is 5 $\times 10^{4} \mathrm{~cm}^{-3}$ and falls below $5 \times 10^{3} \mathrm{~cm}^{-3}$ beyond a distance of $1.5 \mathrm{pc}$; the decrease in density may be described by $n_{\mathrm{H}_{2}} \propto r^{x}$, with $-2 \leq x \leq-1.5$; and the total mass of the core is $10^{4} M_{\odot}$.

The W3 core is collapsing at a rate of a few $\mathrm{km} \mathrm{s}^{-1}$ with a velocity law $v \propto r^{x}$, where $x \geq 0$. Where the foreground $\mathrm{H}$ II regions encounter the molecular core (at its southeast edge), shock fronts precede the ionization fronts into the molecular gas. Since the shocked gas is found to be only a small fraction of the gas in the molecular core, the shock is not responsible for triggering the collapse of the entire core.

The explanation for the overall dynamics and ages of the components in the W3-W4 complex involves a combination of a galactic shock plus a shock associated with the ionization front on the outer edge of W4.

The average relative abundances in the $\mathrm{W} 3$ cloud are $\left[\mathrm{H}_{2}\right] /\left[{ }^{13} \mathrm{CO}\right]=1.3 \times 10^{6}$, with $\left[{ }^{12} \mathrm{CO}\right] /\left[{ }^{13} \mathrm{CO}\right]$ $=40,[\mathrm{CS}] /\left[\mathrm{H}_{2}\right]$ in the range $(2-5) \times 10^{-9}$, and $[\mathrm{HCN}] /\left[\mathrm{H}_{2}\right]$ in the range $(3-8) \times 10^{-9}$. If the analysis of the $\mathrm{HCN}$ data is in error, then the following abundances become possible: $\left[\mathrm{H}_{2}\right] /\left[{ }^{13} \mathrm{CO}\right]=0.9$ $\times 10^{6}$, with $\left[{ }^{12} \mathrm{CO}\right] /\left[{ }^{13} \mathrm{CO}\right]=89$ and $[\mathrm{CS}] /\left[\mathrm{H}_{2}\right]=2$ $\times 10^{-8}$. This would lower molecular hydrogen densities and the mass for the W3 core by a factor of 1.4.

The drop in the CS intensity toward IRS 5 is attributed to a reduction in the relative abundance of CS by as much as a factor of 15-20. The most promising explanation for this decrease involves ionmolecule chemistry which produces variations in the relative abundances of molecules with increasing hydrogen density. 


\section{b) Evolution of the W3 Molecular Cloud}

The following scenario summarizes the model of the W3 region developed from a combination of our observations and the theoretical calculations of Woodward (1978), Gerola and Glassgold (1978), Yorke and Krügel (1977), Elmegreen and Lada (1977), and Bodenheimer, Tenorio-Table, and Yorke (1979). (All protostars in this discussion refer to developing $\mathrm{O}$ and B stars only.) The reader is referred to Figures $3 a$ and $3 b$ for the locations of the features discussed below.

Roughly $4 \times 10^{7}$ years ago an interstellar cloud with a mass $\gtrsim 5 \times 10^{4} M_{\odot}$ (and radius $\gtrsim 40 \mathrm{pc}$ ) entered the density-wave shock of the Perseus spiral arm. Second, $4 \times 10^{6}$ years ago this cloud was squeezed and distorted such that at the head of the cloud, a fragment with a density of around $400 \mathrm{~cm}^{-3}$ became unstable and began to collapse. About the same time, another fragment of lower density $\left(\sim 100 \mathrm{~cm}^{-3}\right)$ further to the rear of the cloud also started to collapse. Third, 2.1 $\times 10^{6}$ years ago the denser lump further fragmented into about 100 protostellar clumps. These individual clumps continued collapsing to form the currently observed $\mathrm{O}$ and B stars of Ocl 352. Fourth, by $1.9 \times 10^{6}$ years ago, when these stars had reached the main sequence and began ionizing the surrounding gas, the second concentration (W3 main) was barely two-thirds through one free-fall time in its collapse. Fifth, $4 \times 10^{5}$ years ago the stars of Ocl 352 had cleared out much of the surrounding gas and the $\mathrm{H}$ II region (W4) began expanding into the remaining molecular cloud. At this time, the W3 main contained a hierarchy of clumps from which about 30 protostars started to collapse over a $2 \times 10^{5}$ year period. The star cluster IRS 5 was the most recent group to begin the final collapse. The protostars associated with the currently observed sources, G133.8+1.4, AFGL 333, and W3(OH), had also started to collapse (possibly triggered by the passage of the shock front of the W4 H II region).
Sixth, since the exciting star(s) of IC 1795 formed closest to the edge of the W3 main molecular cloud, $1 \times 10^{5}$ years ago, its developing $\mathrm{H}$ II region became the first to reach this edge. From then to the present, the various protostars of the $\mathrm{W} 3$ main have and are still reaching the main sequence and developing $\mathrm{H}$ II regions. Seventh, by $1 \times 10^{4}$ years ago IC 1795 had pretty well expanded and ionized the gas of the molecular cloud in the direction of the observer, while the compact $\mathrm{H}$ II region $\mathrm{W} 3 \mathrm{~A}$ was just encountering the edge of the W3 core (which is all that remains of the W3 main molecular fragment). Eighth, today a small jet of optical emission (including [O III] and $\mathrm{H} \alpha$ emission) is seen associated with W3A, indicating that it is just breaking through the edge of the W3 molecular core (Dickel 1979; Dickel and Harten 1980). Ninth, fifty thousand years from now, the more embedded sources such as W3C should break out of the W3 core. Tenth, by that time the southern group of $\mathrm{H}$ II regions should have fully emerged and compact $\mathrm{H}$ II regions should be developing around the IRS 5 cluster. Perhaps as soon as $10^{5}$ years from now, all the stars which formed out of the original W3 main fragment will appear as a loose cluster with their $\mathrm{H}$ II regions merged into one and very little remaining of the original molecular cloud.

This research was partially supported by the National Science Foundation (AST 77-21021) and by the Sterrewacht te Leiden during my most enjoyable stay there. Special thanks go to Teije de Jong for the use of his radiative transfer program and to Paul Woodward for elucidating his galactic shock calculations. The many discussions with various Leiden astronomers during the developmental stages of the model were most helpful. I wish to thank John Dickel, Bill Wilson, and Linda DeNoyer for their suggestions and improvements to the manuscript.

\section{REFERENCES}

Allen, C. W. 1973, Astrophysical Quantities, (3d ed.; London: Athlone Press), p. 209.

Bernes, C. 1979, Astr. Ap., 73, 67.

Bodenheimer, P., Tenorio-Tagle, G., and Yorke, H. W. 1979, Ap. J., 223, 85 .

Brandt, J. C. 1960, Ap. J., 131, 293.

Courtès, G. 1960, Ann. Ap., 28, 683.

de Jong, T., Chu, S.-I., and Dalgarno, A. 1975, Ap. J., 199, 69.

Dickel, H. R. 1979, in IAU Symposium No. 87, Interstellar Molecules, Mont Tremblant, Quebec, August 6-10, 1979.

Dickel, H. R., Dickel, J. R., Wilson, W. J., and Werner, M. W. 1980, Ap. J., 237, $711\left(\mathrm{D}^{2} \mathrm{WW}\right)$

Dickel, H. R., and Harten, R. 1980, in preparation.

Dickman, R. L. 1976, Ph.D. thesis, Columbia University.

. 1978, Ap. J. Suppl., 37, 407.

Elmegreen, B. G., and Lada, C. J. 1977, Ap. J., 214, 725.

Genzel, R., and Downes, D. 1977, Astr. Ap. Suppl., 30, 145.

Georgelin, Y. M., and Georgelin, Y. P. 1976, Astr. Ap., 49, 57.

Gerola, H., and Glassgold, A. E. 1978, Ap. J. Suppl., 37, 1.

Goldreich, P., and Kwan, J. 1974, Ap. J., 189, 441 .

Gottlieb, C. A., Lada, C. J., Gottlieb, E. W., Lilley, A. E., and Litvak, M. M. 1975, Ap. J., 202, 655.
Green, S., and Chapman, S. 1978, Ap. J. Suppl., 37, 169.

Green, S., and Thaddeus, P. 1974, Ap. J., 191, 653.

Hackwell, J. A., Gehrz, R. D., Smith, J. R., and Briotta, D. A. 1978, Ap. J., $221,797$.

Hagen, G. 1970, Pub. David Dunlap Obs., 4, 1.

Harten, R. 1980 , in preparation.

Hartquist, T. W., Oppenheimer, M., and Dalgarno, A. 1980, Ap. J., 236, 182.

Hayford, P. 1932, Lick Obs. Bull., 16, 53.

Henning, K. 1979, Ph.D. thesis, Hamburg Observatory.

Humphreys, R. M. 1976, Ap. J., 206, 114.

Iglesias, E. 1977, Ap. J., 218, 697.

Iglesias, E., and Silk, J. 1978, Ap. J., 226, 851.

Ishida, K. 1969, M.N.R.A.S., 144, 55.

1970, Pub. Astr. Soc. Japan, 22, 277.

Kwan, J. 1978, Ap. J., 223, 147.

Lada, C. J., Elmegreen, B. G., and Blitz, L. 1979, in Protostars and Planets, ed. T. Gehrels (Tucson: University of Arizona Press).

Lada, C. J., Elmegreen, B. G., Cong, H.-Ih., and Thaddeus, P. 1978, Ap. J. (Letters), 226, L39.

Lang, K. R. 1974, Astrophysical Formulae (New York: SpringerVerlag), p. 278. 
Leung, C. M. 1878, Ap. J., 225, 427.

Liszt, H. S., and Leung, C. M. 1977, Ap. J., 218, 396.

Lucas, R. 1976, Astr. Ap., 46, 473.

Mitchell, G. F., Ginsburg, J. L., and Kuntz, P. J. 1978, Ap. J. Suppl., 38,39 .

Ogura, K., and Ishida, K. 1976, Pub. Astr. Soc. Japan, 28, 651

Palouš, J., Ruprecht, J., Dluzhnevskaya, O. B., and Piskunov, T. 1977, Ast. Ap., 61, 27.

Panagia, N. 1973, Ap. J., 78, 929.

Roberts, W. W. 1972, Ap. J., 173, 259

Sanford, R. 1949, Ap. J., 110, 117.

Schmidt, M. 1965, in Stars and Stellar Systems, Vol. 5, ed. A. Blaauw and M. Schmidt (Chicago: University of Chicago Press), p. 513.

Scoville, N. Z., and Solomon, P. 1974, Ap. J. (Letters), 187, L67.

Spitzer, L. 1968, Diffuse Matter in Space (New York: Interscience).
Stark, A. A., and Blitz, L. 1978, Ap. J. (Letters), 225, L15.

Stothers, R. 1972, Ap. J., 175, 431.

Sullivan, W. T., III, and Downes, D 1973, Astr. Ap., 29, 369.

Underhill, A. B. 1967, in IAU Symposium No. 30, Determination of Radial Velocities and Their Applications, ed. A. H. Batten and J. F. Heard (New York: Academic Press), p. 30.

Wendker, H. J., and Altenhoff, W. J. 1977, Astr. Ap., 54, 301.

Westbrook, W. E., Werner, M. W., Elias, J. H., Gezari, D. Y. Hauser, M. G., Lo, K. Y., and Neugebauer, G. 1976, Ap. J., 209, 94.

White, R. E. 1977, Ap. J., 211, 744

Woodward, P. R. 1976, Ap. J., 207, 484.

- 1978, in IAU Symposium No. 84, Large Scale Characteristics of the Galaxy, ed. W. B. Burton (Dordrecht: Reidel), in press. Yorke, H. W., and Krügel, E. 1977, Astr. Ap., 54, 183.

HÉLÈNE R. Dickel: Astronomy Department, University of Illinois, 341 Astronomy Building, Urbana, IL 61801 